\title{
Structure and Magnetism of GaMnN Nanowires Synthesized with Nonmagnetic Catalyst
}

\author{
Zheng Ma, Michelle E. Jamer, Eugen Panaitescu, Don Heiman and Latika Menon \\ Department of Physics, Northeastern University, Boston, MA 02115
}

\begin{abstract}
We demonstrate the synthesis of Mn-doped GaN nanowires by the vapor-liquid-solid (VLS) mechanism, wherein Mn-doping was initiated through a nonmagnetic Au catalyst and chemical vapor deposition (CVD). Structural and morphological characterization studies using X-ray diffraction and electron microscopy confirm the doping of $\mathrm{Mn}$ in wurzite GaN nanowires with a concentration of 0.2 to $3 \%$. The magnetic measurements indicate a combination of room temperature ferromagnetism, plus a paramagnetic component due to antiferromagnetic superexchange.
\end{abstract}

Keywords: Nanowires, chemical vapor deposition, room temperature ferromagnetism, dilute magnetic semiconductors

\section{Introduction}

Dilute magnetic semiconductors (DMS) are of great interest due to their potential applications in spintronic devices [1, 2]. DMS are semiconductors doped with magnetic or rare earth ions and thus exhibit strong magnetic properties. Mn doping of III-V based semiconductors, such as GaMnAs, exhibit ferromagnetism due to the $p-d$ exchange coupling of the Mn spin-5/2 moments by the itinerant holes from the $\mathrm{Mn}$ acceptor. [3] Mn-doped GaN was predicted to be a DMS with a Curie temperature $\mathrm{T}_{\mathrm{C}}>300$ K. However, recent studies have shown that substitution of $\mathrm{Mn}$ on the Ga sublattice has been limited to $\sim 10 \% \mathrm{Mn}$ leading to a maximum $\mathrm{T}_{\mathrm{C}} \sim 13 \mathrm{~K}$. [1] Previous studies have shown successful Mn doping of GaN nanowires [4-15], however, controlling the nanowires properties including morphology, growth direction and composition remains a challenge.

Previous growth of Mn-doped GaN nanowires employed Ni as a catalyst in the VLS [16] method [5, 7, 9$11,13,15]$. However, the Ni catalyst is itself ferromagnetic. In the present study, Au catalyst was used since it is a nonmagnetic substance widely used in VLS. Mn was added either from the reaction source (in vapor state) or from the catalyst (liquid state). A correlation was found between the way in which $\mathrm{Mn}$ is added to the reaction (catalyst versus source) and the growth morphology (serrated versus nonserrated) and growth direction ([0001]vs [1010]) of the nanowires. This is the first demonstration of $\mathrm{Mn}$-doped $\mathrm{GaN}$ wires wherein Mn-doping has been initiated through the catalyst. The main advantages of this method are that, (1) it is amenable to large-scale synthesis of GaMnN with using a magnetic catalyst, and (2) the amount of Mn-doping is controllable depending on the application. This study focuses on the structural and magnetic properties of the Mn-doped GaN nanowires using the Mn-Au alloy as a catalyst.

(C) 2015. This manuscript version is made available under the Elsevier user license 


\section{Experimental Details}

GaMnN nanowires were prepared using the CVD method, with Au as catalyst, previously deposited on the Si substrate by e-beam evaporation. Gallium oxide powder (which sublimates at the temperatures used for nanowire growth) was used as reaction source carried a mixture of ammonia (the $\mathrm{N}$ source) and hydrogen gas with flow rates of 30 and 50 standard cubic centimeters per minute $(\mathrm{sccm})$, respectively. Growth time was typically 1 hour. In the first set of experiments, the manganese source was $\mathrm{MnO}_{2}$ vapor, from powder placed upstream next to the $\mathrm{Ga}_{2} \mathrm{O}_{3}$ powder. The growth temperature varied in the range of $800-1000^{\circ} \mathrm{C}$, at which $\mathrm{Au}$ is in its liquid phase. In the second set of experiments, prior to the CVD runs, metallic Mn was successively deposited on $\mathrm{Si}(100)$ substrates by thermal evaporation after the Au deposition. Growth temperature was maintained at $1000^{\circ} \mathrm{C}$ and pressure inside the quartz tube was maintained at 100 Torr.

Scanning electron microscopy (SEM) and energy dispersive spectroscopy (EDS) were used to obtain images and compositional information using a Hitachi S-4800 SEM system. A JEOL 2010F transmission electron microscope (TEM) was used to obtain high resolution images of single wires. X-ray diffraction (XRD) $\theta-2 \theta$ measurements were carried out using PANalytical Xray X'Pert Pro Diffractometer using a Cu$K \alpha$ radiation source. The magnetic measurements were taken using a Quantum Design superconducting quantum interface device (SQUID) magnetometer. The sample was positioned parallel to the applied magnetic field direction inside the SQUID magnetometer. Though the general wire growth direction is vertical to the substrate, the wire alignment is expected to be random with respect to the magnetic field direction.

\section{Results}

\section{A. Microscopy and composition}

The structure and composition of the nanowires is very different for the two sets of experiments we conducted. In the first case, when $\mathrm{Mn}$ was added to the reaction from $\mathrm{MnO}_{2}$ vapor, the preferred morphology was a serrated one, involving periodical change of the diameter along the nanotubes length, as previously reported $[17,18]$. Figure $1(a)$ shows SEM images of the serrated nanowires produced under these conditions, with a hexagonal cross-section. Electron diffraction spectroscopy (EDS) measurements did not detect any $\mathrm{Mn}$ in the composition of nanowires. This is consistent with previously reported XRD measurements on this type of wires, where no shift in the GaN peak positions was observed. However, $\mathrm{MnO}_{2}$ vapor presence appears to favor and stabilize this type of morphology. The ratio of serrated nanowires to straight ones is increasing with increasing $\mathrm{MnO}_{2}$ addition in the reaction chamber [18]. Also, longer serrated nanowires (over $10 \mu \mathrm{m}$ ) are observed, with constant amplitude and wavelength of the periodical serrations preserved over the whole length of the nanowires.

Lattice-resolved TEM imaging (Fig. 1b) revealed that the nanowires are single crystals, with the growth direction along the polar axis [0001]. The measured values for lattice parameters $a=0.278 \mathrm{~nm}$ and $c=$ $0.52 \mathrm{~nm}$ are typical for the GaN wurzite structure. The selected area electron diffraction (SAED) pattern recorded at the $[12 \overline{3} 0]$ zone axis (Fig. 1b) further confirmed the growth direction and the lattice 
parameters dimensions. These measurements are in agreement with the results obtained in a previous study [5] of serrated nanowires synthesized without addition of $\mathrm{MnO}_{2}$.

A second VLS method using the binary Au-Mn catalyst successfully incorporated $\mathrm{Mn}$ into the GaN nanowires. Most of the nanowires synthesized this way are straight (Figure 1c), with a triangular crosssection. Lattice-resolved TEM imaging (Figure 1d) indicates that the resulting GaMnN wires are growing

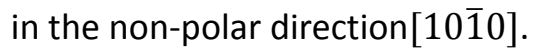
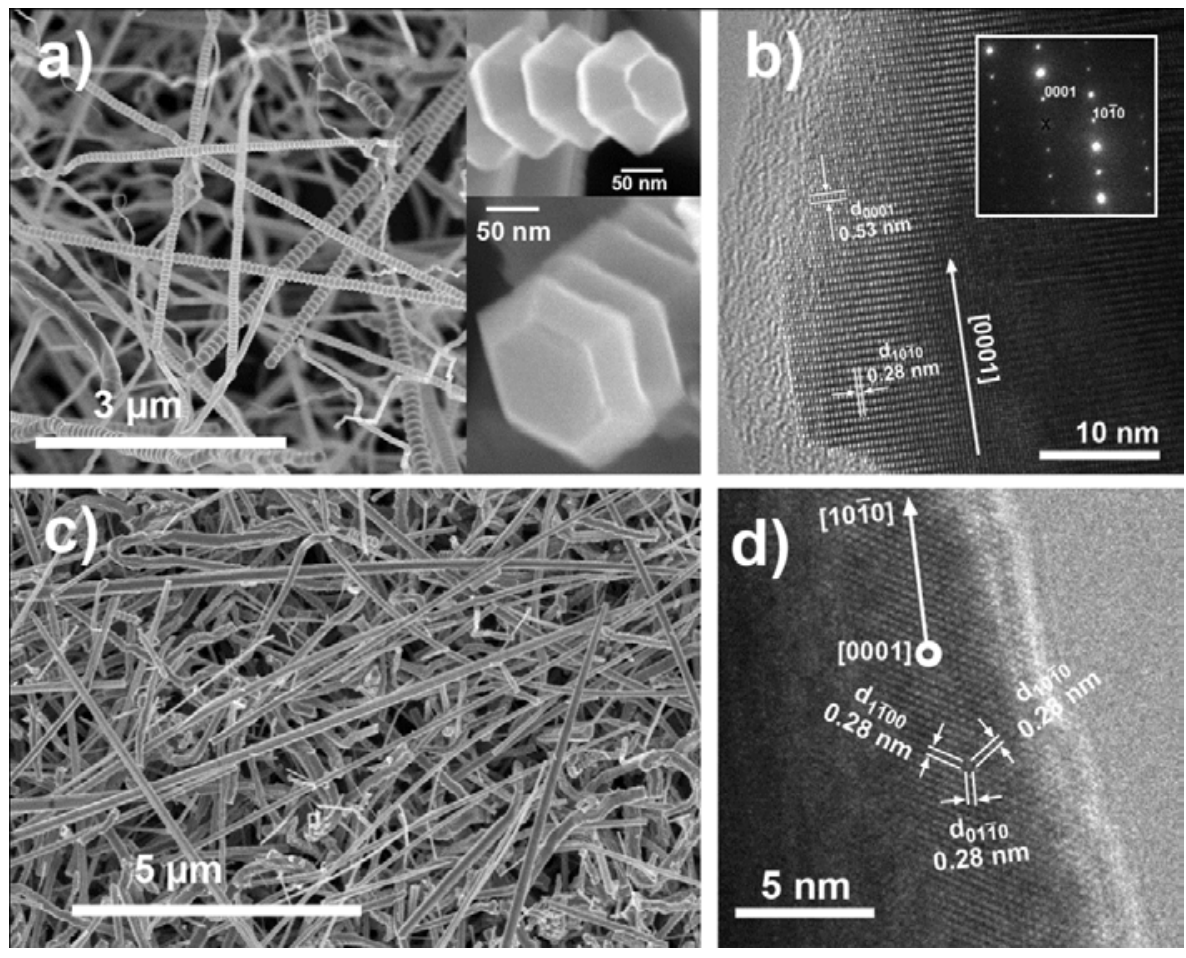

FIG. 1. (a) SEM image of mostly serrated GaN nanowires grown by VLS-CVD with $\mathrm{MnO}_{2}$ added in vapor state. High magnification insets are showing the periodic serration and hexagonal cross-section. (b) Lattice-resolved HRTEM of a single nanowire, with the corresponding SAED pattern in the inset. (c) SEM image of mostly straight GaMnN nanowires grown by VLS-CVD with Au-Mn alloy catalyst. (d) Latticeresolved HRTEM image of a single nanowire revealing a hexagonal pattern with interplanar distances corresponding to the $\{10 \overline{1} 0\}$ family of planes.

Both EDS and XRD were used to confirm successful doping of GaMnN nanowires. EDS spectra were obtained for two types of samples, (1) single GaMnN nanowires, and (2) arrays of GaMnN nanowires attached to the Si substrate. When single nanowires were measured, the atomic percentage of $\mathrm{Mn}$ in GaMnN was estimated to be in the range of $0.15-0.75 \%$, which can vary based on natural variations between nanowires. However, measurement of the GaMnN nanowires still attached to the substrate led to a larger $\mathrm{Mn}$ concentration, on the order of $3 \% \mathrm{Mn}$. This discrepancy is due to the additional contribution from the unused $\mathrm{Mn}$ in the $\mathrm{Mn}-\mathrm{Au}$ alloy catalyst. Based on EDS analysis of many wires, the average percentage of $\mathrm{Mn}$ is estimated to be $\sim 0.5 \%$. 


\section{B. X-ray diffraction}

XRD was also used to estimate the concentration of $\mathrm{Mn}$ in the GaMnN nanowires. GaN has a wurzite structure with a space group of $P 6_{3} m c$ and lattice occupations $G a @ 2 b(1 / 3,2 / 3,0)$ and $N @(1 / 3,2 / 3$, 3/8). The bulk GaN and undoped GaN nanowires have lattice parameters $a=3.189$ and $3.183 \AA$ and $c=5.185$ and $5.190 \AA$, respectively $[19,20]$. From the XRD pattern in Fig. 2, lattice parameters of $a=3.192$ $\pm 0.016 \AA$ and $c=5.184 \pm 0.104 \AA$ were calculated. Previous studies indicate that the $a$-lattice constant increases and the $c$-lattice constant decreases as the $\mathrm{Mn}$ concentration increases [20]. For a $\mathrm{Mn}$ concentration between 0.5-1\%, the lattice constants would be $a=3.187$ and $c=5.189 \AA$. The computed lattice constants are consistent with the average $\sim 0.5 \% \mathrm{Mn}$ incorporated into the GaN lattice.


FIG. 2. (a) XRD pattern of GaMnN with respective Bragg peaks marked with vertical (red) lines. Au is marked with a dotted (purple) line and Ga is marked with a dashed (blue) line. (b) Williamson-Hall plot for the Bragg peaks of the GaMnN nanowires. It is seen that there is a large amount of strain in the lattice. 
In addition to the strong Bragg peaks in the XRD pattern from the hexagonal GaMnN, Bragg peaks were seen corresponding to Ga and Au. The (152) and (153) Bragg peaks of the Ga orthorhombic structures were identified. There were also cubic Au peaks found in the XRD pattern including the (200) and (222) Bragg peaks. These Bragg peaks had much lower intensities. There were no $\mathrm{Mn}$-related peaks found in the XRD pattern. At the particular ratio of $\mathrm{Mn} / \mathrm{Au}$, the sample would display either $\mathrm{\gamma}-\mathrm{Mn}$ or $\alpha-\mathrm{Mn}$ Bragg peaks [21]. Also, we did not observe any XRD peaks associated with $\mathrm{Mn}_{\mathrm{x}} \mathrm{Ga}$, which could contribute a ferromagnetic response [22].

Rietveld analysis showed that $98 \%$ of the material in the XRD pattern was GaMnN [23, 24]. The structure was evaluated by calculating the chemical structure order parameter

$$
S=V\left[I_{(101)}^{m} / I_{(102)}^{m}\right] / V\left[I_{(101)}^{c} / I_{(102)}^{c}\right]
$$

where $\mathrm{I}^{\mathrm{m}}$ and $\mathrm{I}^{\mathrm{c}}$ are the measured and calculated intensities, respectively, of the (101) and (102) Bragg peaks $[25,26]$. The calculation showed that chemical ordering is $S=0.80$. The Williamson-Hall plot shown in the inset of Fig. 2 uses Young's modulus to determine the crystallite size (D) and strain $(\varepsilon)$ of the hexagonal nanowires. There are anisotropic deformations included in the strain in the hexagonal crystal structure [27]. Therefore, Young's modulus is computed with respect to the deformation energy $u=$ $\varepsilon^{2} Y_{h k l} / 2$. This equation is valid for the assumption that the density is uniform. The equation for Young's modulus is [28]

$$
Y_{h k l}=\frac{\left[h^{2}+(h+2 k)^{2} / 3+(a * l / c)^{2}\right]^{2}}{s_{11}\left(h^{2}+(h+2 k)^{2} / 3\right)^{2}+s_{33}(a l / c)^{4}+\left(2 s_{13}+s_{44}\right)\left(h^{2}+(h+2 k)^{2} / 3\right)(a l / c)^{2}}
$$

where $(h k l)$ are the Miller indices, $s_{i j}$ are the elastic compliance coefficients. When Young's modulus is incorporated into the Williamson-Hall plot, the equation becomes

$$
\beta_{h k l} \cos \theta=\frac{K \lambda}{D}+u^{1 / 2}\left(4 \sin \theta\left(\frac{2}{Y_{h k l}}\right)^{1 / 2}\right)
$$

The Scherrer constant was assumed to be $K=1, \lambda=0.15418 \mathrm{~nm}$, and instrumental broadening in the Bragg $2 \theta$ angle was neglected. The average strain was calculated to be $1.2 \%$ and $D=88 \mathrm{~nm}$. These values are attributed to possible atom dislocations in the wurzite structure due to the relief of strain [29, 30]. The TEM results indicated that the $\mathrm{Mn}$ concentration varies along the nanowires, with more $\mathrm{Mn}$ existing at the lower portion of the wires. It is possible that strain can also affect the lattice constants in the wurtzite structure, indicating that $\mathrm{Mn}$ concentration cannot reliably be extracted through XRD. The XRD results indicated that there was excess $A u$ and $G$ a after the formation of GaMnN nanowires and there were no peaks being attributed to $\mathrm{Mn}$, which indicates that there are no $\mathrm{Mn}$ or $\mathrm{Mn}-\mathrm{Au}$ nanoclusters on the substrate which are detectable by XRD. 


\section{Magnetic Properties}

The magnetic properties of the doped GaMnN nanowires obtained in the second set of experiments were measured as a function of both temperature and applied magnetic field. Figure 3(a) shows magnetization $(\mathrm{m})$ versus field curves in the temperature range $10-300 \mathrm{~K}$. There are two components, a ferromagnetic (FM) signal that saturates at low fields, and large paramagnetic (PM) signal that grows at lower temperatures.
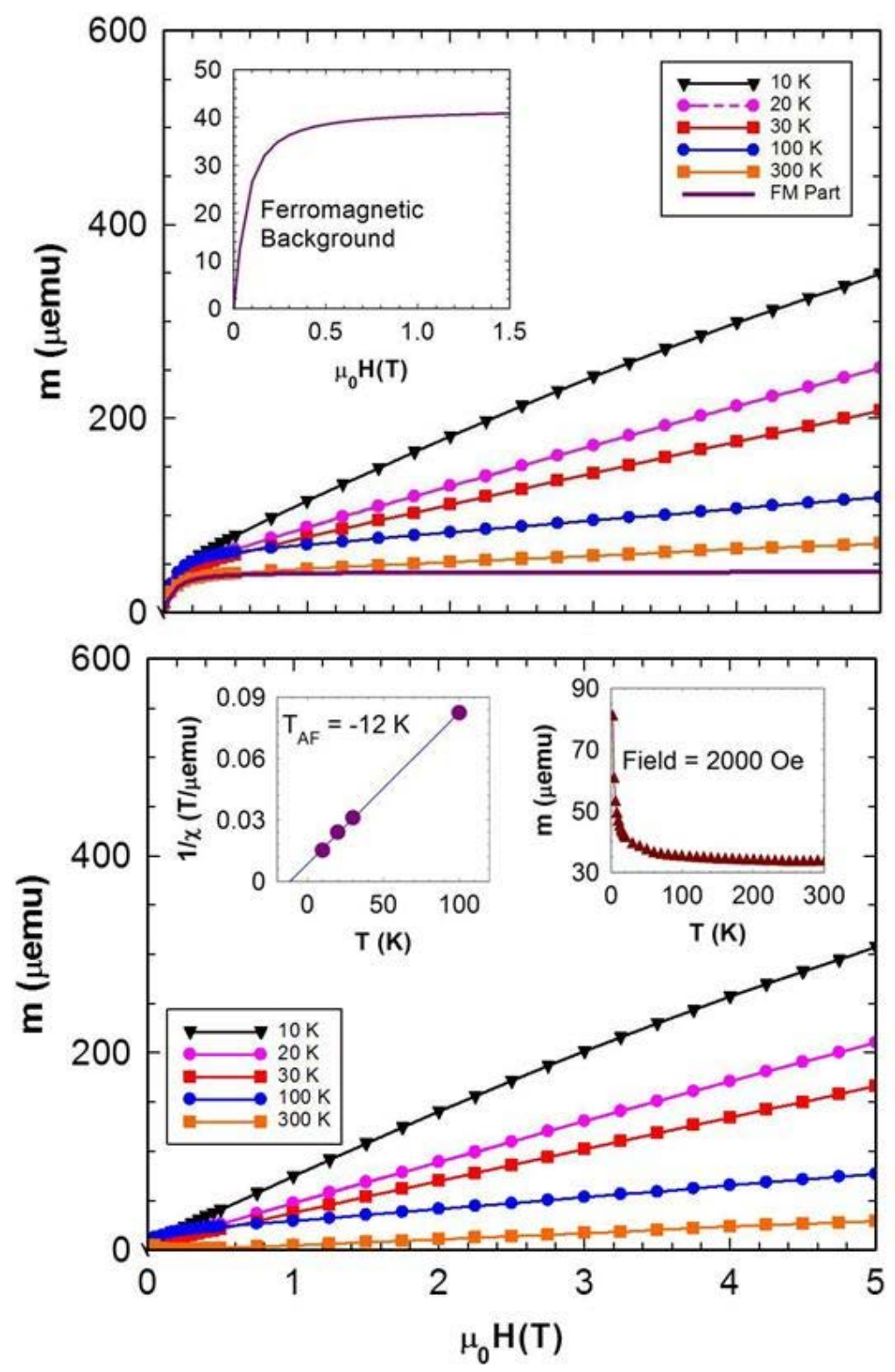

FIG. 3. (a) Magnetic moment (m) versus applied magnetic field of the $G a_{1-x} M n_{x} N(\langle x\rangle=0.005)$ nanowires. The inset shows the FM component of $m$, obtained after subtracting the PM component. (b) PM component of the magnetic moment after subtracting the FM component. The right inset shows $m(T)$ following a Curie-Weiss law. The left inset shows that the Weiss constant is negative, indicating a negative AF exchange interaction. 
The inset of Fig. 3(a) shows the temperature independent FM component that saturates by $0.5 \mathrm{~T}$. Upon subtraction of this FM component from the total $\mathrm{m}$, Fig. 3(b) indicates a linear dependence typical of PM [31]. The right inset in Fig. 3b shows $\mathrm{m}(\mathrm{T})$ taken at 2000 Oe, characteristic of a Curie-Weiss law for PM. The left inset in Fig. 3(b) shows the susceptibility versus temperature, indicating the Weiss constant $\mathrm{T}_{\mathrm{AF}}=$ $-12 \mathrm{~K}$. The negative value indicates AF exchange interactions between a subset of $\mathrm{Mn}$ ions [32]. The cause of the high temperature FM in dilute ferromagnetic semiconductors is controversial. The majority of the sample is GaMnN as seen in Rietveld refinement, therefore it is unlikely the joint PM and FM signals are due to Au-Mn or $\mathrm{Mn}$ nanoclusters. However, it is possible that there are small amounts of nanoclusters of $\mathrm{Mn}_{\mathrm{x}} \mathrm{Ga}$ or Mn-rich precipitates, which are not detected by XRD that could lead to the high-temperature FM.

There are several explanations for the high temperature FM component - a second phase of $\mathrm{Mn}_{\mathrm{x}} \mathrm{Ga}$ or a carrier-mediated effect where the nanostructuring leads to a large concentration of holes in the nanowires. The experimental evidence of Curie temperatures in $\mathrm{GaMn}_{x} \mathrm{~N}$ near room temperature is quite controversial [1, 33]. Recently, careful experimental studies have let to conclude that for $\mathrm{Mn}$ concentrations approaching $x \sim 0.1$ the Curie temperature reaches only $T_{C} \sim 13 \mathrm{~K}$ [34]. These results are consistent with a tight-binding model of superexchange interactions and Monte Carlo simulations of $\mathrm{T}_{\mathrm{C}}$. Thus, for our concentrations where $x<3 \%$ the superexchange mechanism is not expected to lead to the observed high-temperature FM. Previous literature has shown that when the zinc-blende nanocrystals are grown at high temperatures, there can be spinodal decomposition [35, 36]. We also note that $\mathrm{Mn}_{\mathrm{x}} \mathrm{Ga}$ has Curie temperatures in the range of 600-800 K [37], so a second phase of $\mathrm{Mn}_{\mathrm{x}} \mathrm{Ga}$ could be present. A carrier-mediated model is less likely to cause the FM but it cannot be ruled out. The nanowires have diameters of $\sim 100 \mathrm{~nm}$ leading to large surface to volume ratio. Defects and impurities on the surface could lead to increased hole densities and $\mathrm{Mn}(2+)$ ions. With a sufficient concentration of itinerant holes the FM coupling between $\mathrm{Mn}$ ions may lead to higher Curie temperatures. Now note that the PM contribution is much larger than the FM contribution. It is known that in the dilute limit of dopants $(x<0.01)$, the $\mathrm{Mn}$ atoms would contribute independently to the magnetism and the Brillouin function can model the PM of the dopants [38, 39]. The observed PM is likely to be caused by the compensated electronic nature of the system, where the nearest-neighbor $\mathrm{Mn}$ ions experience AF superexchange, leading to the observed negative $T_{A F}=-12 \mathrm{~K}$ [40]. Also, as-synthesized $\mathrm{GaN}$ is normally n-type with a large concentration of electrons [41] $\left(\sim 10^{16-18} \mathrm{~cm}^{-3}\right)$, which can compensate the number of holes from the $\mathrm{Mn}$ acceptors.

\section{Summary}

A correlation was found between the manner in which $\mathrm{Mn}$ is introduced in the VLS-CVD reaction and the resultant morphology and growth direction of GaN nanowires. In the present method using an Au-Mn alloy catalyst, $\mathrm{Mn}$ dopants were introduced into $\mathrm{GaN}$ resulting in single crystal wurtzite $\mathrm{GaMnN}$ nanowires with $\mathrm{Mn}$ concentrations up to $3 \%$. XRD and EDS confirmed that $\mathrm{Mn}$ was integrated into the GaN nanowires. Two magnetic components were observed: a quickly saturating temperatureindependent FM moment was present, with a high Curie temperature $T_{C}>400 \mathrm{~K}$; and a strongly 
temperature-dependent PM component. The FM could be associated with $\mathrm{Mn}_{\mathrm{x}} \mathrm{Ga}$ regions or regions having larger $\mathrm{Mn}$ concentrations and itinerant carriers, while the PM is associated with regions dominated by antiferromagnetic superexchange.

\section{Acknowledgements}

We thank T. Hussey for assistance with the SQUID magnetometer. The work was supported by the U.S. National Science Foundation grants ECCS-0925285, DMR-0907007 and ECCS-1402738. M.E.J. would like to acknowledge the support of the International Centre for Diffraction Data's Ludo Frevel Scholarship.

\section{References}

[1] T. Dietl, H. Ohno, Dilute ferromagnetic semiconductors: Physics and spintronic structures, Reviews of Modern Physics, 86 (2014) 187-251.

[2] I. Žutić, J. Fabian, S. Das Sarma, Spintronics: Fundamentals and applications, Reviews of Modern Physics, 76 (2004) 323-410.

[3] T. Dietl, H. Ohno, F. Matsukura, J. Cibert, D. Ferrand, Zener Model Description of Ferromagnetism in Zinc-Blende Magnetic Semiconductors, Science, 287 (2000) 1019-1022.

[4] Y.-K. Byeun, K.-S. Han, H.-J. Choi, S.-C. Choi, Controlled doping of single crystalline diluted magnetic semiconductor Ga1-xMnxN nanowires, Materials Science and Engineering: A, 452-453 (2007) 499-502.

[5] H.J. Choi, H.K. Seong, J. Chang, K.I. Lee, Y.J. Park, J.J. Kim, S.K. Lee, R. He, T. Kuykendall, P. Yang, Single-Crystalline Diluted Magnetic Semiconductor GaN:Mn Nanowires, Advanced Materials, 17 (2005) 1351-1356.

[6] F.L. Deepak, P.V. Vanitha, A. Govindaraj, C.N.R. Rao, Photoluminescence spectra and ferromagnetic properties of GaMnN nanowires, Chemical Physics Letters, 374 (2003) 314-318.

[7] S.S. Farvid, M. Hegde, I.D. Hosein, P.V. Radovanovic, Electronic structure and magnetism of Mn dopants in GaN nanowires: Ensemble vs single nanowire measurements, Applied Physics Letters, 99 (2011) 222504.

[8] D.S. Han, J. Park, K.W. Rhie, S. Kim, J. Chang, Ferromagnetic Mn-doped GaN nanowires, Applied Physics Letters, 86 (2005) -.

[9] T.-H. Kim, C.-O. Jang, H.-K. Seong, H.-J. Choi, S.-K. Lee, Fabrication and Electrical Characterization of Heterojunction Mn-Doped GaN Nanowire Diodes on n-Si Substrates (GaN:Mn NW/n-Si), Journal of Elec Materi, 38 (2009) 505-510.

[10] E. Oh, J.H. Choi, H.-K. Seong, H.-J. Choi, Optical properties of GaN and GaMnN nanowires grown on sapphire substrates, Applied Physics Letters, 89 (2006) 092109.

[11] P.V. Radovanovic, C.J. Barrelet, S. Gradečak, F. Qian, C.M. Lieber, General Synthesis of Manganese-Doped II-VI and III-V Semiconductor Nanowires, Nano Letters, 5 (2005) 1407-1411.

[12] M.L. Reed, N.A. El-Masry, H.H. Stadelmaier, M.K. Ritums, M.J. Reed, C.A. Parker, J.C. Roberts, S.M. Bedair, Room temperature ferromagnetic properties of (Ga, Mn)N, Applied Physics Letters, 79 (2001) 3473-3475.

[13] M. Ren, H. Huang, H. Wu, D. Zhao, H. Zhu, Y. Liu, B. Sun, Growth of high quality GaN nanowires by using $\mathrm{Ga} / \mathrm{GaCl} 3$ sources, Physica E: Low-dimensional Systems and Nanostructures, 57 (2014) 145-148.

[14] G. Thaler, R. Frazier, B. Gila, J. Stapleton, M. Davidson, C.R. Abernathy, S.J. Pearton, C. Segre, Effect of Mn concentration on the structural, optical, and magnetic properties of GaMnN, Applied Physics Letters, 84 (2004) 1314-1316.

[15] C. Xu, J. Chun, H.J. Lee, Y.H. Jeong, S.-E. Han, J.-J. Kim, D.E. Kim, Ferromagnetic and Electrical Characteristics of in Situ Manganese-Doped GaN Nanowires, The Journal of Physical Chemistry C, 111 (2007) 1180-1185.

[16] R.S. Wagner, W.C. Ellis, VAPOR - LIQUID - SOLID MECHANISM OF SINGLE CRYSTAL GROWTH, Applied Physics Letters, 4 (1964) 89-90.

[17] Z. Ma, D. McDowell, E. Panaitescu, A.V. Davydov, M. Upmanyu, L. Menon, Vapor-liquid-solid growth of serrated GaN nanowires: shape selection driven by kinetic frustration, Journal of Materials Chemistry C, 1 (2013) 7294-7302. [18] A. Patra, Z. Ma, L. Menon, A.V. Gopal, Optical properties of serrated GaN nanowires, Opt. Mater. Express, 4 (2014) 1373-1382.

[19] M. Leszczynski, H. Teisseyre, T. Suski, I. Grzegory, M. Bockowski, J. Jun, S. Porowski, K. Pakula, J.M. Baranowski, 
C.T. Foxon, T.S. Cheng, Lattice parameters of gallium nitride, Applied Physics Letters, 69 (1996) 73-75.

[20] S. Sonoda, H. Hori, Y. Yamamoto, T. Sasaki, M. Sato, S. Shimizu, K.-i. Suga, K. Kindo, Properties of ferromagnetic $\mathrm{Ga}_{1-x} \mathrm{Mn}_{\mathrm{x}} \mathrm{N}$ films grown by ammonia-MBE, Magnetics, IEEE Transactions on, 38 (2002) 2859-2862.

[21] B. Predel, Numerical Data and Functional Relationships: Phase Equilibria, Crystallographic and Thermodynamic Data of Binary Alloys: Ac-Au... Au-Zr, Landolt-Börnstein, 1991.

[22] M.E. Jamer, B.A. Assaf, S.P. Bennett, L.H. Lewis, D. Heiman, Magnetic properties and large coercivity of MnxGa nanostructures, Journal of Magnetism and Magnetic Materials, 358-359 (2014) 259-262.

[23] L.B. McCusker, R.B. Von Dreele, D.E. Cox, D. Louer, P. Scardi, Rietveld refinement guidelines, Journal of Applied Crystallography, 32 (1999) 36-50.

[24] H. Rietveld, A profile refinement method for nuclear and magnetic structures, Journal of Applied Crystallography, 2 (1969) 65-71.

[25] C.T. Chantler, Detailed Tabulation of Atomic Form Factors, Photoelectric Absorption and Scattering Cross Section, and Mass Attenuation Coefficients in the Vicinity of Absorption Edges in the Soft X-Ray (Z=30-36, Z=60-89, $\mathrm{E}=0.1 \mathrm{keV}-10 \mathrm{keV})$, Addressing Convergence Issues of Earlier Work, Journal of Physical and Chemical Reference Data, 29 (2000) 597-1056.

[26] H.P. Hanson, F. Herman, J.E. Lea, S. Skillman, HFS atomic scattering factors, Acta Crystallographica, 17 (1964) 1040-1044.

[27] Y. Rosenberg, V.S. Machavariani, A. Voronel, S. Garber, A. Rubshtein, A.I. Frenkel, E.A. Stern, Strain energy density in the x-ray powder diffraction from mixed crystals and alloys, Journal of Physics: Condensed Matter, 12 (2000) 8081.

[28] V. Mote, Y. Purushotham, B. Dole, Williamson-Hall analysis in estimation of lattice strain in nanometer-sized ZnO particles, Journal of Theoretical and Applied Physics, 6 (2012) 6.

[29] B.D. Cullity, S.R. Stock, Elements of x-ray diffraction, Prentice Hall, Upper Saddle River, 2001.

[30] G.K. Williamson, W.H. Hall, X-ray line broadening from filed aluminium and wolfram, Acta Metallurgica, 1

(1953) 22-31.

[31] M. Cohen, Introduction to Magnetic Materials, Addison-Wesley, Boston, MA, 1972.

[32] A. Van Esch, L. Van Bockstal, J. De Boeck, G. Verbanck, A.S. van Steenbergen, P.J. Wellmann, B. Grietens, R. Bogaerts, F. Herlach, G. Borghs, Interplay between the magnetic and transport properties in the III-V diluted magnetic semiconductor $\mathrm{Ga}_{1-\mathrm{x}} \mathrm{Mn}_{\mathrm{x}} \mathrm{As}$, Physical Review B, 56 (1997) 13103-13112.

[33] G.T. Thaler, M.E. Overberg, B. Gila, R. Frazier, C.R. Abernathy, S.J. Pearton, J.S. Lee, S.Y. Lee, Y.D. Park, Z.G. Khim, J. Kim, F. Ren, Magnetic properties of n-GaMnN thin films, Applied Physics Letters, 80 (2002) 3964-3966.

[34] S. Stefanowicz, G. Kunert, C. Simserides, J.A. Majewski, W. Stefanowicz, C. Kruse, S. Figge, T. Li, R. Jakieła, K.N. Trohidou, A. Bonanni, D. Hommel, M. Sawicki, T. Dietl, Phase diagram and critical behavior of the random ferromagnet Ga\$\{\}_\{1\ensuremath\{-\}x\}\$Mn\$\{\}_\{x\}\$N, Physical Review B, 88 (2013) 081201.

[35] A. Bonanni, Ferromagnetic nitride-based semiconductors doped with transition metals and rare earths, Semiconductor Science and Technology, 22 (2007) R41.

[36] T. Dietl, Origin of ferromagnetic response in diluted magnetic semiconductors and oxides, Journal of Physics: Condensed Matter, 19 (2007) 165204.

[37] M.E. Jamer, B.A. Assaf, T. Devakul, D. Heiman, Magnetic and transport properties of Mn2CoAl oriented films, Applied Physics Letters, 103 (2013) 142403.

[38] J.K. Furdyna, Diluted magnetic semiconductors, Journal of Applied Physics, 64 (1988) R29-R64.

[39] D. Heiman, E.D. Isaacs, P. Becla, S. Foner, High-field magnetization of (Cd,Mn)Te, Physical Review B, 35 (1987) 3307-3310.

[40] E.D. Isaacs, D. Heiman, X. Wang, P. Becla, K. Nakao, S. Takeyama, N. Miura, Spin saturation of the random antiferromagnet (Cd,Mn)Te in magnetic fields to 150 T, Physical Review B, 43 (1991) 3351-3355.

[41] J.D. Albrecht, R.P. Wang, P.P. Ruden, M. Farahmand, K.F. Brennan, Electron transport characteristics of GaN for high temperature device modeling, Journal of Applied Physics, 83 (1998) 4777-4781. 